University of Wollongong

Research Online

Faculty of Engineering and Information

Faculty of Engineering and Information

Sciences - Papers: Part A

Sciences

2012

\title{
Nanoscale characterization of ion tracks: MC simulations versus analytical approach
}

\author{
M U. Bug \\ University of Wollongong, mb355@uowmail.edu.au \\ E Surdutovich \\ Oakland University \\ H Rabus \\ Physikalisch-Technische Bundesanstalt Germany \\ Anatoly B. Rosenfeld \\ University of Wollongong, anatoly@uow.edu.au
}

A V. Solov'Yov

Frankfurt Institute for Advanced Studies

Follow this and additional works at: https://ro.uow.edu.au/eispapers

Part of the Engineering Commons, and the Science and Technology Studies Commons

Research Online is the open access institutional repository for the University of Wollongong. For further information contact the UOW Library: research-pubs@uow.edu.au 


\title{
Nanoscale characterization of ion tracks: MC simulations versus analytical approach
}

\author{
Abstract \\ The goal of this work was to investigate the agreement of physical parameters related to the particle \\ track structure on the nanometer scale, obtained by means of a detailed track structure simulation (PTra) \\ as well as by a rapid analytical approach. Parameters describing the tracks of secondary electrons \\ produced by $0.3 \mathrm{MeV} / \mathrm{u} \mathrm{C} 6+$-ions were of particular interest as those particles are densely ionizing. For \\ this purpose, the target volume in form of a nanometric water cylinder was positioned at different radial \\ distances from the ion trajectory and track structure parameters were determined as function of the radial \\ distance. While the fluence of electrons through the target surface and the mean ionization cluster size \\ obtained by both approaches were in good agreement, the probabilities of specific cluster sizes (one, two \\ and three) turned out to be rather different in the two approaches.

\section{Keywords} \\ versus, analytical, approach, nanoscale, ion, characterization, tracks, mc, simulations \\ Disciplines \\ Engineering | Science and Technology Studies

\section{Publication Details} \\ Bug, M. U., Surdutovich, E., Rabus, H., Rosenfeld, A. B. \& Solov'Yov, A. V. (2012). Nanoscale \\ characterization of ion tracks: MC simulations versus analytical approach. The European Physical Journal \\ D, 66 (11), 291-296.
}




\title{
Nanoscale characterization of ion tracks: MC simulations versus analytical approach
}

\author{
Marion U. Bug ${ }^{1,2}$, Eugene Surdutovich ${ }^{3,4}$, Hans Rabus ${ }^{1}$, Anatoly B. Rosenfeld ${ }^{2}$, and Andrey V. Solov'yov ${ }^{4}$ \\ 1 Physikalisch-Technische Bundesanstalt (PTB), Bundesallee 100, 38116 Braunschweig, Germany \\ 2 Centre for Medical Radiation Physics, University of Wollongong, Wollongong NSW 2522, Australia \\ 3 Department of Physics, Oakland University, Rochester, Michigan 48309, USA \\ 4 Frankfurt Institute for Advanced Studies (FIAS), Ruth-Moufang-Str. 1, 60438 Frankfurt am Main, Germany
}

Received: date / Revised version: date

\begin{abstract}
The scope of this work was to investigate the agreement of physical parameters related to the particle track structure on the nanometer scale, obtained by means of a detailed track structure simulation (PTra) as well as by a rapid analytical approach. Parameters describing the tracks of secondary electrons produced by $0.3 \mathrm{MeV} / \mathrm{u} \mathrm{C}^{6+}$-ions were of particular interest as those particles are densely ionizing. For this purpose, the target volume in form of a nanometric water cylinder was positioned at different radial distances from the ion trajectory and track structure parameters were determined as function of the radial distance. While the fluence of electrons through the target surface and the mean ionization cluster size obtained by both approaches were in good agreement, the probabilities of specific cluster sizes (one, two and three) turn out to be rather different in the two approaches.
\end{abstract}

\section{Introduction}

The biological effects of ionizing radiation are commonly accepted to be initiated by DNA damage, where the different effectiveness of different radiation qualities is attributed to their potential of creating DNA damage of high complexity (e.g. a high number of strand breaks within a short segment of the DNA) [1]. This potential is related to the microscopic particle track structure, which particulary has to be taken into account for densely ionizing radiation, such as heavy charged particles.

The particle track structure can be characterized by physical parameters, such as the probability distribution of ionization cluster size or the electron fluence. The ionization cluster size is defined as the number of direct ionizations produced by a single incident particle of specific type and energy (and its secondaries) within a specified volume, which is typically chosen to be equal in size to one DNA convolution [2]. This quantity is used in nanodosimetry where it is related to the number of DNA strand breaks $[2,3]$. The electron fluence, on the other hand, was used within the framework of a multiscale approach to the physics of ion-beam cancer therapy [4]. In this approach, the assessment of DNA damage was based on the number of secondary electrons incident onto the surface of a volume, corresponding to one DNA convolution, using a parameter deduced from biological experiments $[4,5]$.

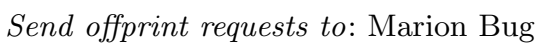

The desired physical parameters of particle track structure can be obtained by special Monte Carlo simulations, in which the transport of all primary and secondary particles is followed event-by-event through the material [6-8]. The drawback of such simulations is that they require a long CPU time as well as the input of detailed interaction cross section data for the simulated medium with the ionizing particles, which are not always available.

An analytical approach has been developed to overcome these limitations of detailed track structure simulations through a fast calculation of the desired physical parameters by accounting only for the most relevant physical phenomena (see section 2.3) [4]. This should facilitate the future use of track structure parameters in practical applications.

Due to the rapid calculation, the analytical approach would be particularly beneficial for determining track structure parameters within the locally well-defined Bragg peak region near the end of an ion track. In this region, a large number of secondary electrons is produced in ionization interactions due to the maximum of the ion's stopping power [9]. Most of these electrons are of low energy (average energy on the order of several tens of eV) and are therefore also densely ionizing within a small region. As the direction of the ions is practically left unchanged by their interactions with target molecules, energy depositions in the vicinity of the ion trajectory are entirely due to secondary electrons $[5,10]$.

This work aims to compare physical parameters of secondary electron track structure, exemplarily for electrons 
produced by $\mathrm{C}^{6+}$-ions with their Bragg peak energy of about $0.3 \mathrm{MeV} / \mathrm{u}$. Those parameters (i.e. the radial dependence of electron fluence and of the distribution of ionization cluster size for a target volume equal to a DNA convolution) were obtained using both track structure simulations and the analytical approach.

\section{Methods}

\subsection{Physical parameters of track structure}

Physical parameters related to the track structure of $\mathrm{C}^{6+}$ ions of $0.3 \mathrm{MeV} / \mathrm{u}$ energy were determined via both the analytical approach and track structure simulations using the dedicated code PTra [7]. An introduction to both methods is given below (sections 2.2 and 2.3).

The target volume, to which those parameters were related, was represented by a water cylinder equal in size to one convolution of the DNA (height $L=3.4 \mathrm{~nm}$ and radius $a=1.15 \mathrm{~nm}$ ), positioned at a radial distance $\rho$ from the ion trajectory (fig. 1).

The first track structure parameter investigated was the fluence ${ }^{1}$ of secondary electrons crossing the surface of the target volume located at a certain distance from the ion trajectory was investigated.

Furthermore, two parameters related to the nanodosimetrical approach developed by Grosswendt et al. [7] were determined, namely the mean ionization cluster size and the probability distribution of cluster sizes. The ionization cluster size $\nu$ is defined as the number of ionizations produced by a single primary particle of specific radiation quality $Q$ (type and energy of a particle, e.g. a 0.3 $\mathrm{MeV} / \mathrm{u} \mathrm{C}^{6+}$-ion) and its secondaries within the specific target volume. A frequency distribution of ionization cluster size, obtained for a high number of incident particles, approximates the statistical distribution of the probability $P(\nu \mid \rho, Q)$ to obtain exactly $\nu$ ionizations within the target volume located at a distance $\rho$ from the ion trajectory. To obtain the probability distribution, a normalization has to be performed according to

$$
\sum_{\nu=0}^{\infty} P(\nu \mid \rho, Q)=1 .
$$

The probability distribution of ionization cluster sizes and derived statistical moments, such as the mean ionization cluster size

$$
M_{1}(\rho, Q)=\sum_{\nu=0}^{\infty} \nu P(\nu \mid \rho, Q)
$$

characterize the particle track and are unique for a specific radiation quality. Furthermore, these quantities can be related to the probability of producing a DSB via models,

\footnotetext{
1 Note that the terms 'fluence' and 'flux' used throughout this manuscript differ from the ICRU definition as they are applied here as synonyms for the number of electrons crossing the surface of the target cylinder in any direction (per time interval, when applicable to the 'flux').
}

such as those proposed by Grosswendt et al. or Garty et al. $[2,11]$.

In the following sections, the track structure simulation by means of PTra and the analytical approach to determine the aforementioned physical parameters are described.

\subsection{PTra simulation}

Monte Carlo codes are useful to simulate the inherent stochastic nature of radiation transport in matter. Track structure simulations were specifically developed to obtain a spatial resolution on the order of the mean free path as they are able to transport particles and their secondaries event-by-event through the matter by treating each interaction with the medium individually until the particle energy falls below a specified threshold [6-8,10,12].

The track structure code PTra (version PTra-h2o-1201) was used, which was specifically developed and is well established for nanodosimetry $[7,11,13]$. A comprehensive description of the particle transport in such kinds of simulations is provided in $[6-8,10,12,14]$ and therefore omitted in this paper. To enable such simulations, interaction cross section data for the medium are required as input parameters. The interaction cross section set for water used in PTra was comprehensively specified in Lazarakis et al. [14]. In the present work an extension of the lower energy threshold from $10.76 \mathrm{eV}$ to $1.7 \mathrm{eV}$ was included to account also for electrons below the ionization threshold, as such electrons would be able to disrupt target molecules by attachment processes. The extension of the threshold was accomplished by taking nine more phonon and vibrational excitation states as well as two dissociative attachment anion states into account, in addition to the five ionization and five electronic excitation levels considered hitherto. The total cross sections for these low-energy excitations were taken directly from Michaud et al. [15] for all energies they measured, i.e. between $1 \mathrm{eV}$ and $100 \mathrm{eV}$. The excitation energy for each level was random sampled from a truncated Lorentzian distribution, as proposed and parameterized by Dingfelder et al. [16]. Angular deviations due to phonon and vibrational excitation were assumed to be the same as for elastic scattering, whereas for dissociative attachment anion states no change in direction was applied.

The setup of the simulation is sketched in fig. 1, with the ion trajectory long enough to include the contribution of all secondary electrons that were able to reach the target volumes (about $60 \mathrm{~nm}$ trajectory length). The target volumes were placed at specific distances $\rho$ from the ion trajectory, starting at the radius of the cylinder of 1.15 $\mathrm{nm}$, such that the target volume just 'touches' the ion trajectory. Also in this case, only ionizations related to secondary electrons were taken into account. To obtain the fluence, the number and direction of electrons crossing the surface of each target cylinder were sampled for the front surface (defined with regard to the origin of the electron along the ion trajectory). The relevant fluence was determined by subtracting the number of electrons leaving 
the cylinder through the front surface from the number of electrons entering the cylinder through the front surface. The probability of ionization cluster size was determined by sampling the number of ionizations within each target volume.

To obtain sufficient statistics, the track structure simulation was repeated for a total number of $10^{4} \mathrm{C}^{6+}$-ions.

\subsection{Analytical approach}

In the current work, a three-dimensional random walk of secondary electrons, produced along the ion track, is used to calculate the fluence through a given target volume. In this analytical approach, the key quantity is the flux of secondary electrons through a patch $d \boldsymbol{A}$ located at a distance $r$ from a segment $d \zeta$ of the ion trajectory from which the secondary electrons originate. It is given by ${ }^{2}$ $[17,18]$ :

$$
\begin{array}{r}
\frac{d^{2} N_{C}(r, t)}{d t d \zeta}=d \boldsymbol{A} \cdot D \nabla P(t, r) \frac{d N}{d \zeta} \\
=d \boldsymbol{A} \cdot D \mathbf{n}_{\mathbf{r}} \frac{\partial P(t, r)}{\partial r} \frac{d N}{d \zeta}
\end{array}
$$

where $D=\bar{v} l / 6$ is the diffusion coefficient, $\bar{v}$ is the average velocity of the electron, $l$ is the mean free path for elastic scattering of electrons in the medium, $\mathbf{n}_{\mathbf{r}}$ is a unit vector in the radial direction (from the segment to the center of the patch $d \boldsymbol{A}$ ),

$$
P(t, r)=\left(\frac{3}{2 \pi \bar{v} t l}\right)^{3 / 2} \exp \left(-\frac{3 r^{2}}{2 \bar{v} t l}\right)
$$

is the probability density to observe a randomly walking electron at a time $t$ and a distance $r$ from the electron's origin, and $\frac{d N}{d \zeta}$ is the number of secondary electrons emitted per $d \zeta$-segment of the ion trajectory.

In order to calculate the fluence through the surface of a target cylinder with its axis perpendicular to the ion trajectory and located at a radial distance $\rho$, eq. (3) was integrated over time and then over $d \boldsymbol{A}$. For the time integration, eq. (4) was substituted in eq. (3) and the variables were changed from $t$ to the number of steps $k$ of the secondary electrons using $\bar{v} t=k l$. This gives

$$
\begin{aligned}
\frac{d N_{C}(r)}{d \zeta}= & \int \frac{d^{2} N_{C}(r, t)}{d t d \zeta} d t=d \boldsymbol{A} \cdot \mathbf{n}_{\mathbf{r}} \frac{d N}{d \zeta} \int_{r / l}^{\infty} d k \\
& \frac{r}{2 k}\left(\frac{3}{2 \pi k l^{2}}\right)^{3 / 2} \exp \left(-\frac{3 r^{2}}{2 k l^{2}}-\gamma k\right) .
\end{aligned}
$$

An exponential attenuation factor $e^{-\gamma k}$ was introduced in eq. (5) in order to take into account electrons lost from the random walk due to inelastic scattering. The constant $\gamma$ is equal to the ratio of the effective cross section of

\footnotetext{
${ }^{2}$ Note that the dependence on the radiation quality $Q$ of the quantities in the equations related to the analytical model were omitted to improve the readability
}

inelastic processes to the total cross section for electron scattering. The integration over $k$ was carried out from the minimal number of steps necessary to reach distance $r$, to infinity. The fluence of electrons through the cylinder surface was obtained by the integration of (5) over $d \boldsymbol{A}$, where the same geometrical setup as in the simulations with PTra was chosen, such that the fluence determined by the means of both approaches could be compared. The coordinates related to the cylinder were chosen, such as $z$ along its axis and $\phi$ as an azimuthal angle (fig. 1). Using

$$
d \boldsymbol{A} \cdot \mathbf{n}_{\mathbf{r}}=a d \phi d z \frac{\rho \cos \phi+\zeta \sin \phi-a}{r},
$$

where $\zeta$ is the coordinate along the ion trajectory and

$$
r=\sqrt{(\rho-a \cos \phi)^{2}+(\zeta-a \sin \phi)^{2}+z^{2}},
$$

the fluence of electrons through the cylinder surfaces is given by the following integral:

$$
\begin{aligned}
& N_{C}(\rho)=\frac{d N}{d \zeta} 4 a \int_{0}^{L / 2} d z \int_{0}^{\infty} d \zeta
\end{aligned}
$$

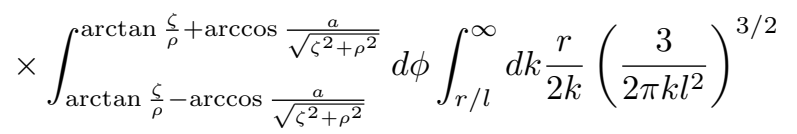

$$
\begin{aligned}
& \times \exp \left(-\frac{3 r^{2}}{2 k l^{2}}-\gamma k\right) \frac{\rho \cos \phi+\zeta \sin \phi-a}{r} .
\end{aligned}
$$

The integration was obtained with an attenuation factor of $\gamma=0.0001$, an elastic mean free path of $l=0.2 \mathrm{~nm}$ and an electron production rate of $d N / d \zeta=15.2 \mathrm{~nm}^{-1}$. The latter value is in accordance with the ionization mean free path of $\mathrm{C}^{6+}$-ions used in the PTra simulations. The value of $l$ corresponds to the elastic mean free path for an electron of about $11 \mathrm{eV}$, which was also found in the PTra simulations as the mean energy of secondary electrons approaching the cylinder surface when the target was located at $1.15 \mathrm{~nm}$. This ensures that the same basic conditions apply to both approaches.

In order to estimate clustered DNA damage using the analytical approach, the mean number of ionizations produced within the target volume by the secondary electrons originating from a single ion was calculated. As an initial attempt, we introduced an effective ionization cross section $\sigma_{\text {eff }}$ in such a way that the probability of ionization of a water molecule inside a cylinder by an electron crossing its lateral surface is equal to the ratio of this cross section and the lateral area of the cylinder $(2 \pi a L)$. Then the average number of ionizations in the cylinder, i.e. the mean ionization cluster size $M_{1}$, is equal to the product of the probability of ionization per electron per molecule $\sigma_{\text {eff }} /(2 \pi a L)$, the number of electrons entering the cylinder $N_{C}$, and the number of water molecules inside the cylinder (number density $n$ times volume $\pi a^{2} L$ ), i.e.,

$$
M_{1}(\rho)=N_{C}(\rho) \frac{n \pi a^{2} L}{2 \pi a L} \sigma_{\mathrm{eff}}(\rho)=N_{C}(\rho) \frac{n a}{2} \sigma_{\mathrm{eff}}(\rho) .
$$


As can be seen from fig. 2, the spectra of secondary electrons passing the target cylinder surfaces extend over several orders of magnitude in energy and vary with increasing distance from the ion trajectory, particularly in the energy range where ionization processes are possible (i.e. for energies exceeding $10.79 \mathrm{eV}$ ). The ionization cross section $\sigma_{\text {ion }}(E)$ varies over several orders of magnitude in this energy range, so that the choice of the appropriate $\sigma_{\text {eff }}(\rho)$ is not straightforward. To get an idea of the magnitude of this quantity and its variation with distance from the ion trajectory, we solved eq. (9) for $\sigma_{\text {eff }}(\rho)$

$$
\sigma_{\text {eff }}(\rho)=\frac{M_{1}(\rho)}{N_{C}(\rho)} \frac{2}{n a}
$$

and inserted the mean cluster size and the fluence obtained by means of PTra simulations as well as the number density $n=33.4 \mathrm{~nm}^{-3}$ corresponding to liquid water of mass density $1 \mathrm{~g} / \mathrm{cm}^{3}$. The resulting values were then fitted by a simple exponential function and compared to the weighted mean of the energy dependent ionization cross section, calculated from the energy spectra at the cylinder surface shown in fig. 2 for electron energies above the ionization threshold of $10.79 \mathrm{eV}$.

The probability distribution of ionization clusters $\nu$ was assumed to follow a Poisson distribution for a specific radial distance $\rho$ from the ion trajectory

$$
P(\nu \mid \rho)=\exp \left(-M_{1}(\rho)\right) \frac{M_{1}(\rho)^{\nu}}{\nu !} .
$$

\section{Results}

Fig. 3 shows the fluence of secondary electrons through the surface of a target cylinder as a function of the distance between the cylinder and the $\mathrm{C}^{6+}$-ion trajectory. The agreement between the analytical approach and the results obtained by PTra is within 15\%, except for distances $\rho>8 \mathrm{~nm}$ from the ion trajectory. The reason for this deviation is most likely due to the limited statistics in the Monte Carlo simulation, as the fluence at these distances, and therefore the number of contributing electrons, is reduced by nearly two orders of magnitude. A major difference between the approaches can be observed, however, for the calculation of the fluence in close vicinity to the ion trajectory. Here, the fluence calculated by the analytical approach shows a build-up effect at small distances (below about $1.3 \mathrm{~nm}$ ). The reason for this is that the lower integration limit over the number of steps in eq. (5) was the minimum number of steps necessary to reach the cylinder surface $(r / l)$. In this approach, there is no contribution of electrons that originate at distances $r$ smaller than $l=0.15 \mathrm{~nm}$, which is the case for a cylinder positioned at $\rho \leq a+l$ (where $a=1.15 \mathrm{~nm}$ is the radius of the target cylinder). In the simulations this effect naturally does not occur.

A similar agreement (within $12 \%$ for distances $\rho<$ $8 \mathrm{~nm}$ from the ion trajectory) between both approaches can also be seen for the mean ionization cluster size in fig. 4. The effective cross section used to determine the mean ionization cluster size differs, however, largely (up to a factor of three) from the one determined by using the energy spectra as is shown in fig. 5. The reason for this discrepancy is due to the assumption of a mean electron energy in the one case, while considering the energy spectra of secondary electrons when calculating the effective ionization cross section in the other case, which is taking into account the large variation of the ionization cross section as function of electron energy. The mean energy of electrons approaching the cylinder surface varies from $11 \mathrm{eV}$ at $\rho=1.5 \mathrm{~nm}$ to about $5 \mathrm{eV}$ at $\rho=10 \mathrm{~nm}$. This means that the elastic mean free path is by several orders of magnitude smaller than the ionization mean free path for the majority of electrons in the energy spectrum. Even if the mean energy is calculated only for electrons of energy above the ionization threshold, this mean value varies from $37 \mathrm{eV}$ at $\rho=1.5 \mathrm{~nm}$ to about $55 \mathrm{eV}$ at $\rho=10 \mathrm{~nm}$. These numbers already indicate the complexity of the electron trajectory and that the assumptions leading to eq. (9) are likely to be too crude.

Another example that the complexity of the electron tracks has to be accounted for in more detail is given by the comparison of radial distributions for probabilities of different ionization cluster sizes, obtained by simulations and according to a Poisson distribution (fig. 6). The maxima of the probabilities obtained analytically exceed those obtained by the simulations by about $30 \%$. The overestimation of small cluster sizes $(\nu=1)$ by the Poisson distribution, together with the underestimation of larger cluster sizes for increasing distances $\rho$, becomes worse with the degree of complexity. This is clearly due to the energy spectrum of secondary electrons extending across three orders of magnitude as well as to the neglect of secondary electrons of low energy, additionally produced by the electrons originating from the ion interaction. It is natural and was also tested in an artificial track structure simulation that the Poisson distribution is valid for monoenergetic electrons, which would not change their mean free path. In this artificial case, the occurrence of large cluster sizes, arising from low-energy electrons in the simulation, as well as the occurrence of smaller cluster sizes (on average), arising from high-energy electrons with a mean free path greater than the one assumed in the analytical model are both suppressed.

\section{Conclusion}

In this work, physical parameters related to the track structure of secondary electrons produced by carbon ions in the Bragg peak region were studied by means of Monte Carlo simulations and an analytical approach. The physical parameters (i.e. the electron fluence, the mean ionization cluster size, and the probability to observe ionization cluster sizes of one, two or three in a cylindrical water volume, representing a single DNA convolution) were investigated as a function of the radial distance between the target volume and the ion's trajectory. These parameters were determined from track structure simulations 
by means of PTra and analytically, promising more rapid calculations. The analytical calculation of the fluence was carried out using a random walk approach, while several additional models were applied to obtain the other quantities.

The fluence and the mean ionization cluster size obtained by both approaches are in satisfactory agreement (within $15 \%$ or $12 \%$, respectively for distances $\rho<8 \mathrm{~nm}$ from the ion's trajectory). It is remarkable that the analytical approach, considering a simple diffusion of secondary electrons by a model which includes just two parameters, is capable of describing the fluence in agreement with Monte Carlo simulations. However, the description of ionization cluster sizes required the introduction of an ionization cross section as function of the distance from the ion's path to the target cylinder. Also, the approximation of the probability distribution of ionization cluster sizes by an ordinary Poisson distribution turned out to be a rather poor estimate. These findings evoke the necessity to complement the random walk approach with a more elaborate model for the production of ionization clusters.

Even though the two approaches were compared at a particular energy of carbon ions, the results are expected to be valid for a large range of ion energies and different types of ions as long as the average energy of secondary electrons remains around $50 \mathrm{eV}$ [4]. However, for more elaborate simulations and analytical models suitable for calculations of ionization clusters, the energy spectrum of secondary electrons is required.

This work lays the foundation for continuing investigations, comparing track structure simulations and analytical methods. The comparison performed here suggests that the analytical approach shows great promise for estimating nanodosimetric quantities rapidly for different sections of an ion track for projectiles of various types and energies. The ongoing collaboration aims to further improve Monte Carlo simulation techniques and the development of more accurate analytical models.

We thank the COST action Nano-IBCT for the stimulation of collaborative links with Australia.

\section{References}

1. D.T. Goodhead, H. Nikjoo, Int. J. Radiat. Biol. 55, 513 (1989)

2. B. Grosswendt, S. Pszona, A. Bantsar, Radiat. Prot. Dosim. 126, 432 (2007)

3. H. Rabus, H. Nettelbeck, Radiat. Meas. 46, 1522 (2011)

4. A.V. Solov'yov, E. Surdutovich, E. Scifoni, I. Mishustin, W. Greiner, Phys. Rev. E 79, 011909 (2009)

5. E. Surdutovich, A. Yakubovich, A.V. Solov'yov, Eur. Phys. J. D 60, 101 (2010)

6. W. Friedland, P. Jacob, H.G. Paretzke, T. Stork, Radiat. Res. 150, 170182 (1998)

7. B. Grosswendt, Radiat. Environ. Biophys. 41, 103 (2002)

8. H. Nikjoo, S. Uehara, W.E. Wilson, M. Hoshi, D.T. Goodhead, Int. J. Radiat. Biol. 73, 355 (1998)

9. U. Amaldi, G. Kraft, Rep. Prog. Phys. 68, 1861 (2005)

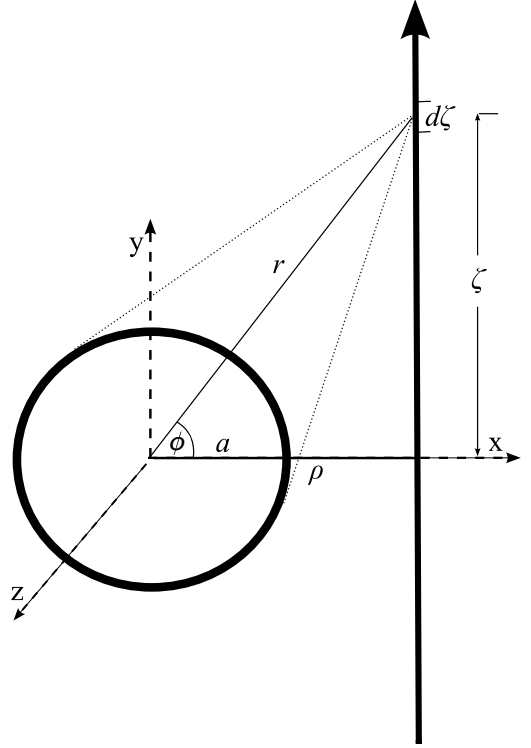

Fig. 1. Sketch of the geometry used in PTra and the analytical model for a $\mathrm{C}^{6+}$-ion beam passing the target cylinder of radius $a$ and length $L$ with an impact parameter $\rho$ (i.e. the distance between the center of the target volume and the ion trajectory).

10. M. Krämer, M. Durante, Eur. Phys. J. D 60, 195 (2010)

11. G. Garty, R. Schulte, S. Shchemelinin, C. Leloup, G. Assaf, A. Breskin, R. Chechik, V. Bashkirov, J. Milligan, B. Grosswendt, Phys. Med. Biol. 55, 761 (2010)

12. S. Incerti, A. Ivanchenko, M. Karamitros, A. Mantero, P. Moretto, H.N. Tran, B. Mascialino, C. Champion, V.N. Ivanchenko, M.A. Bernal et al., Med. Phys. 37, 4692 (2010)

13. R.W. Schulte, A.J. Wroe, V.A. Bashkirov, G.Y. Garty, A. Breskin, R. Chechik, S. Shchemelinin, E. Gargioni, B. Grosswendt, A.B. Rosenfeld, Z. Med. Phys. 18, 286 (2008)

14. P. Lazarakis, M.U. Bug, E. Gargioni, S. Guatelli, H. Rabus, A.B. Rosenfeld, Phys. Med. Biol. 57, 1231 (2012)

15. M. Michaud, A. Wen, L. Sanche, Radiat. Res. 159, 3 (2003)

16. M. Dingfelder, A. Travia, R.A. McLawhorn, J.L. Shinpaugh, L. Toburen, Rad. Phys. Chem. 77, 1213 (2008)

17. E. Surdutovich, D.C. Gallagher, A.V. Solov'yov, Phys. Rev. E 84, 051918 (2011)

18. S. Chandrasekhar, Rev. Mod. Phys. 15, 1 (1943) 


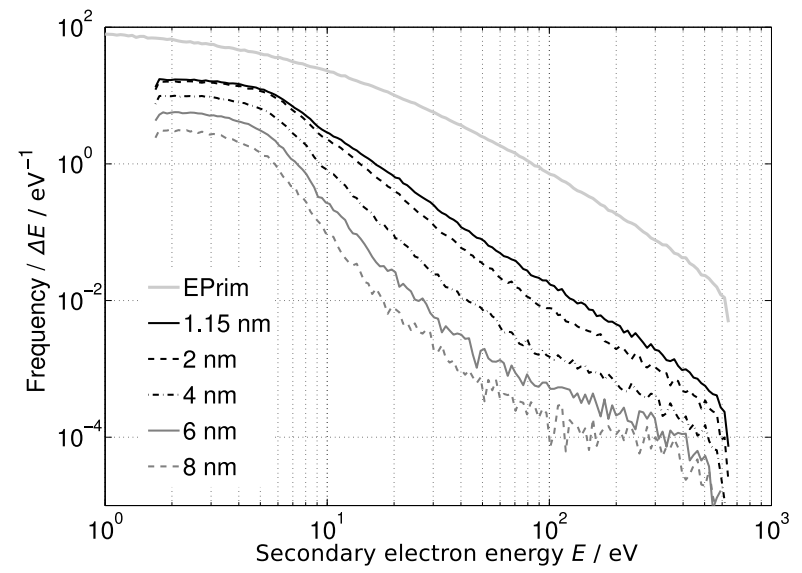

Fig. 2. Energy spectra of electrons directly produced by the ion (EPrim) and of secondary electrons crossing the front surface of a cylinder with its axis located at the indicated distance $\rho$ to the ion trajectory. These data were obtained by means of PTra simulations.

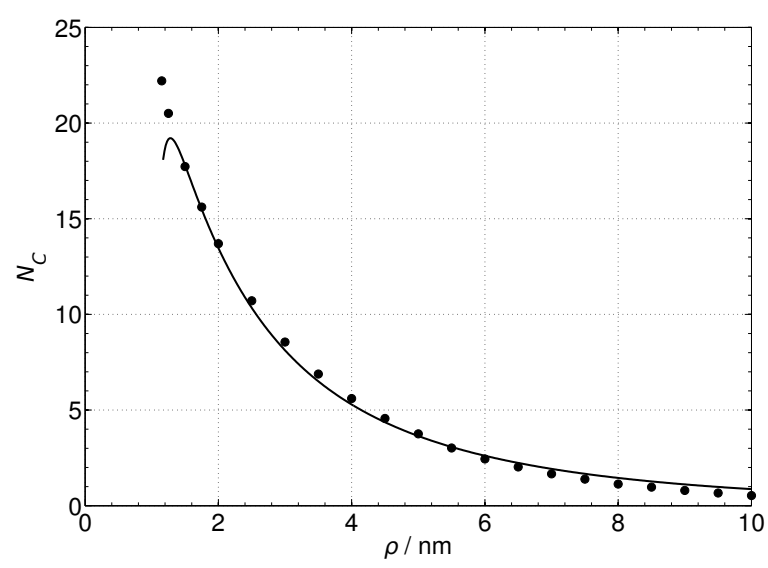

Fig. 3. Fluence of secondary electrons hitting the surface of the cylinder as a function of the distance from the ion's path determined by the analytical approach according to eq. (8) (solid line) and PTra simulations (symbols). Remark: in all figures, the statistical uncertainties of the data obtained by the Monte Carlo simulation do not exceed the symbol size.

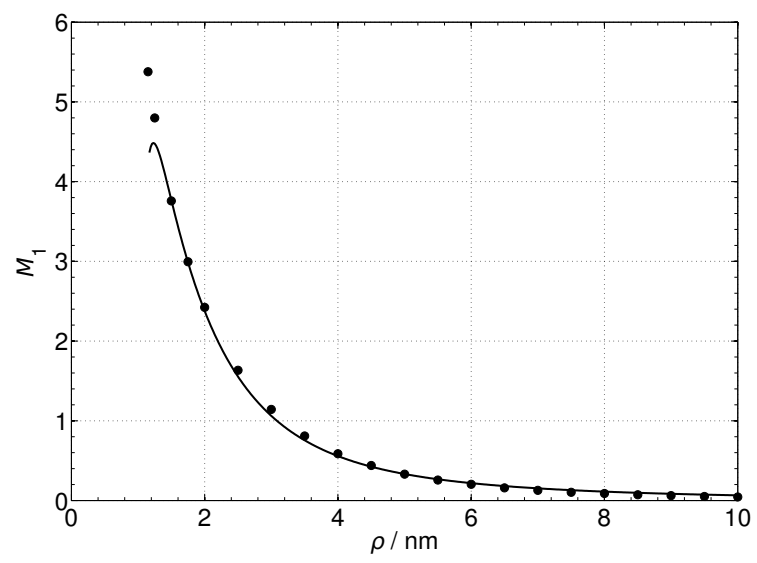

Fig. 4. Mean ionization cluster size produced within the target volume as a function of the distance from the ion trajectory determined by the analytical approach according to eq. (9) (solid line) and PTra simulations (symbols).

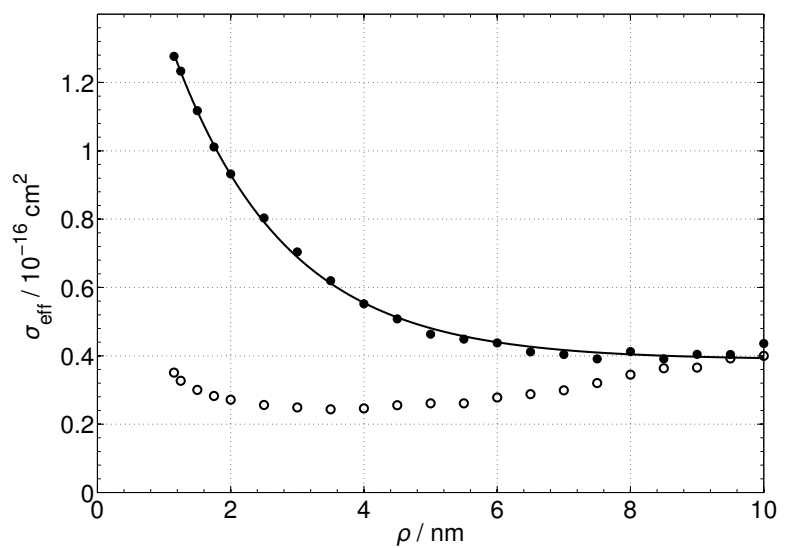

Fig. 5. Effective ionization cross section determined from the simulation according to eq. (10) (filled circles), fit to those data (solid line) by the function $1.762 \cdot 10^{-16} \mathrm{~cm}^{2}$. $\exp \left(-0.5898 \mathrm{~nm}^{-1} \rho\right)+0.3886 \cdot 10^{-16} \mathrm{~cm}^{2}$ and obtained from the energy spectra (open circles) as a function of the distance from the ion track. 


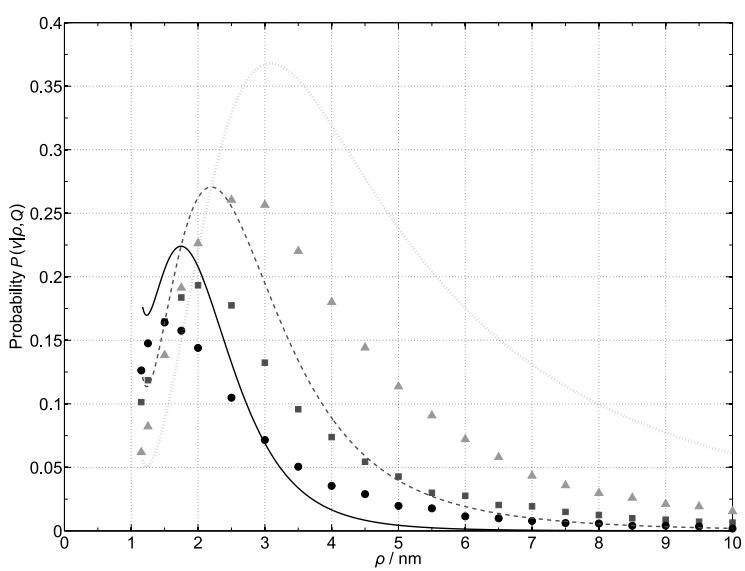

Fig. 6. Probabilities for observing ionization clusters of one (triangles, dotted line), two (squares, dashed line), and three (circles, solid line) as a function of the distance from the ion trajectory determined by the analytical approach according to eq. (11) (lines) and PTra simulations (symbols). 